\title{
Seismic Theory of Tides; Theory of Forced Seismic Waves
}

\author{
Heyfetz Eduard \\ State University of Odessa, named after I. I. Mechnikov, Yaffo, Israel
}

\begin{abstract}
Proceeding from the statement about presence of the normal resilient medium in the cosmic space, the author concludes seismic nature of tides and a number of other aspects of this phenomenon. The analysis of contradiction of the theory of tsunami to empiric facts led the author to conclude that the tsunami are forced and not free waves, and that the key moment in their distribution is the seismic compression of water.
\end{abstract}

Keywords: free waves, forced waves, microseisms, normal resilient medium, tides, tsunami

\section{Introduction}

I will begin the story from the Universe. Currently the Big Bang is accepted as being the beginning of Universe. Nevertheless, we know that explosion may proceed in different finite volumes-grenade, star, etc., -whereas its application to the Universe is contradictory to classical philosophy would require further proof. As far as I know, it was reduced to self-conviction by replacement of the term of "Meta-galaxy" with "Universe".

All that we really know about the Universe is that it is united. As I will show later, there is no void. Therefore, such unity is not formal but physical. At the same time, the Universe is composed of galaxies, stars, planets, humanity, etc. Hence the question follows: Why does it not represent the only existing basic unit—a clot of the being?

The explanation perhaps is that the being is characterized by greatness. This implies that it should be greater than anything existing inside it - and this lesser part should be separated in reality, i.e., physically. Because any part of the being is great by itself, it should be greater than its own parts. Hence, both the Universe and its subdivisions are infinite and, furthermore, unlimited (without boundaries). Thus, when our hand touches a table, the distance between them equals to 0 centimetres, millimetres, microns and so on, up to the level of the given smallness, where a transitional zone, common to both hand and table, exists. The finite object in effect has foreign contacts, whereas the Universe is deprived of them.

From the infinity, the unity of the being follows as well as the contrary of this unity - multitude. Each component of the being is divided. Such duality leads to uninterrupted fight between unity and multitude, the individual and its elements (the spontaneous actions of the environment) are realized through movement.

The conclusion about the infinite nature of any being contradicts the paradigm of the elementary particles. The latter does not follow from mere empirical facts. In his Nobel lecture, Dirac (1933) said: "there are other kinds which have not been shown to be composite and which one expects will never be shown to be composite, so

Heyfetz Eduard, independent researcher, M.A. of Science, State University of Odessa, named after I. I. Mechnikov, Yaffo, Israel. 
that one considers them as elementary and fundamental" (Dirac, 1933, p. 320). Such an approach, coming to the ancient atomism, apparently explains the same chemical and physical properties of substances even outside the Earth. Hence, the same sizes of particles and intuitive conclusion they are found are on the most basic level.

Nevertheless, during the struggle between the unity and multitude, the latter tends to equalize the sizes of its components. Such a peculiarity belongs to the philosophical foundations of physics. Thus, the grains of sand in a given locality or drops of rain at a given time are near in size. The spontaneous processes of evolution led to the similar sizes of the living being of a given affinity. The standards of measurement emerged as a result of spontaneous processes in the market, i.e., the approximately equal sizes of particles may be explained by huge spontaneous processes, preceding the Great Bang. Nevertheless, as the author suggests, the Universe is infinite and outside of our meta-galaxy there are substances that are not composed of electrons, protons, and neutrons. In such a way, micro-particles may be divisible, such as "atoms", grains of sand, drops of rain, etc.

According to the accepted formula of division to infinity, $\lim _{y \rightarrow \infty} x: y=0$, the final result of such division would be meaningless. Nevertheless, this formula is of applied character. Hence, it does not deal with infinity, but with applied notion of an innumerable amount.

The limit to an infinitely divided object is not mere infinite, but the infinite totality of its components and only them.

Dividing such an object physically, we would obtain a given volume of an absolutely unstructured matter. It would be impossible to study it physically, for existing equipment has limited sensitivity.

However, it is possible to measure it otherwise, accepting infinity for a definite amount. In this case, we obtain $\lim \mathrm{x}: \infty=\mathrm{x}: \infty_{\mathrm{x} \neq \mathrm{y}}=\mathrm{x}: \mathrm{x}=1_{\mathrm{e}}$, where $1_{\mathrm{e}}$ is the elementary or infinitely small unit, which is indivisible further.

From the infinity, the function, determining co-existence and incoincidence of its components, follows. Infinity suggests at least one direction. Let us then add infinitely small units to a given one. As a result, we would obtain two contrary directions. The point is that co-existence is mutual. Not only does the considered unit co-exist with the added one, but also the added one with the first one. Hence, totality of two mutually defined contrary directions or dimension.

Units are impenetrable: No unit could penetrate the other one to form one unit that equals to the previous two. The same is true for real volumes. Hence, abstracting from the matter, we will obtain its most basic properties.

The real values (bodies, particles, etc.) are separated from totality via movement. The moving unit would divide unidimensional totality into the rear and the fore sub-totalities. It cannot enter in the fore unit; and it cannot shift all the totality of units. In order to allow the considered unit to pass, the fore one should move aside. Hence, the unit in the second dimension represents a square with zero boundaries of two orders.

It is remarkable that the infinitely small units that had a prototype and a mathematical atom were rejected at the end of 17th century. The use of an atom caused difficulties, first and foremost, in geometry. Thus, Kepler did not succeed to express sectors of a circle in atoms. Cavaglieri used atoms to calculate a pyramid volume. In order to avoid grave errors, he was forced to apply different methods. 
Trying to express an angle in infinitely small units, I found that it represents by itself a two-dimensional totality. Lines composing it should, therefore, possess the second dimension or be unidimensional boundary of two-dimensional bases. Accepting the width of a line for infinitely small units, we will find that it is dissected in the apex. This means exaggeration of the internal content, hence, absence of curves and oblique lines, and hence, curved space on the basic level. The observed bending of light near stars is the result of gradual diffraction. Such a phenomenon with its corresponding explanation was predicted by Newton in the case, where there is no void (Newton, Opticks ${ }^{1}$, p. 350 (query 20)).

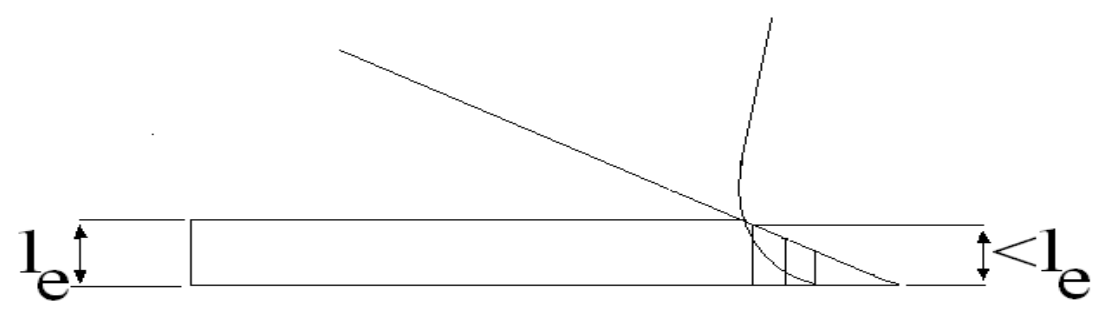

Figure 1. A section of the line, composing an angle.

The same conclusion emerged from the paradox of Democritus (1970), who argued the existence of many cone sections. I argue that the paradox may be reduced to a single section. Let us place the cone section parallel to the base. Are its surfaces equal to one another? If they are equal, then the parts close to the base are not different from those close to the apex, thus, forming a cone cylinder. If they are not equal, the cone cannot be created anew. Instead of a smooth side surface, a step would appear (Democritus, 1970, p. 240).

In practice, we do not confuse cone with a cylinder. On the other hand, by idealizing shapes, we constantly ignore slight unevenness, i.e., the second alternative appears reliable. Nevertheless, the paradox will actually be relative to each single step. Hence, the cone would be divided into cylindrical steps. Applying the same reasoning to longitudinal sections, one will receive a totality of cubes, in which volume is measured.

That is the solution for the infinitely small level. In practice, on an infinitely composed level, there is no basic cube that forms all others. There are no ideal smooth surfaces. On the infinitely composed level, surfaces are exchangeable in transitional self-identical regions. The display of dimensions here is not static, but a dynamic one. There are six infinitely composed directions, forming three dimensions, in which the totality of all movements occurs. Hence, the dimensions by themselves are fluid, therefore, space, composed of them cannot be void.

In a two-dimensional totality, the maximal width of the moving value would equal to $2_{\mathrm{e}}$ infinitely small units. Otherwise, the units found it opposite that its middle cannot pass from the fore sub-totality to the rear one. On the one hand, the real value is infinitely composed, i.e., sufficiently greater than $2_{\mathrm{e}}$.

\footnotetext{
${ }^{1}$ The book was released with the orthography of the XVIII century.
} 


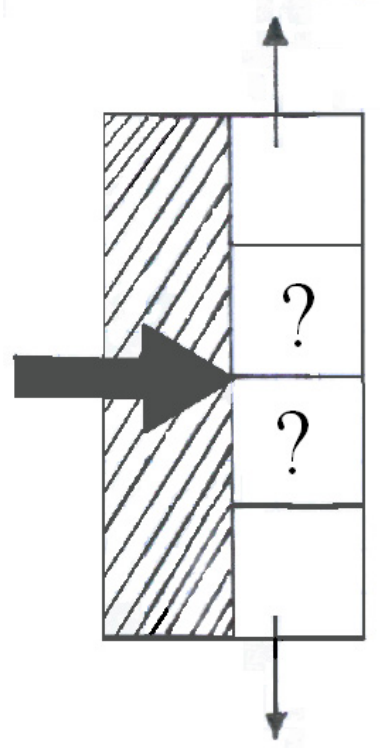

Figure 2. The problem of the two-dimensional totality.

Thus, on the infinitely small level, the third dimension provides the necessary condition for the movement of a value, wider than $2_{\mathrm{e}}$.

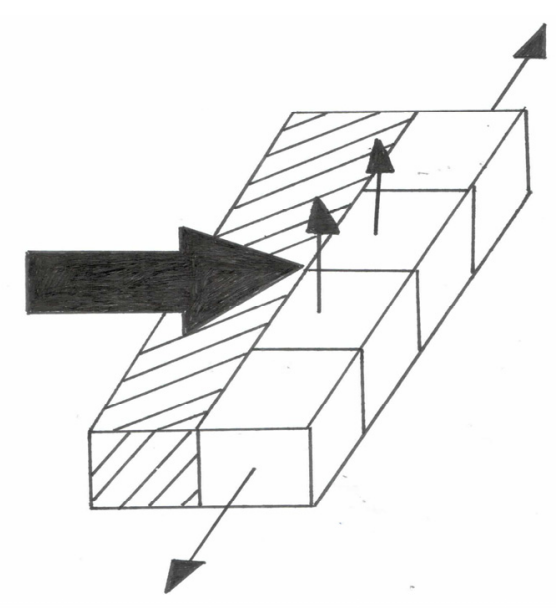

Figure 3. Conclusion of the third dimension.

Nevertheless, the third dimension is infinitely composed as well, i.e., sufficiently greater than 2 e. This means that no moving object may circumvent a given one. Nevertheless, the lesser values penetrate the given one without destructing it, which would be impossible in a two-dimensional totality. Thus, any moving object, 
including micro particles, is represented by a penetrable net from the proper structures on infinite totality of levels.

Therefore, the first dimension represents the direction of the movement of a given value; the second dimension, the being of the value; and the third, the way for the values of lesser significance. It is likely that the number of dimensions is manifested by these conditions. It may be that the author missed some causes for additional dimensions. It could be also noted that the most flattened organisms have tentacles or a bending of the body in the third dimension. The same would be true if the additional ones would exist.

\section{Brief Review of Concepts of the Remote Action}

The first concept of the remote action was given by Descartes (1989), who introduced a notion of thin medium, called ether or gravitational matter that possesses a monopoly on gravity. It may be compared with such imponderable matters as caloric or sound liquids in the physics of XVIII - middle of XIX century. Orbital motion he explained by closed currents of a medium - the so called "whirls"-Descartes emphasized that he means any circulatory motion of matter (Descartes, 1989, p. 392).

Newton was departed from excessive particularization of gravity mechanism in the Cartesian (Descartian) school. He claimed: "Hypotheses non fingo"-_I don't invent hypotheses". Nevertheless, he introduced towards with forces of gravity such a hypothetical factor, as tangential motion of planets. Taking into account that during orbital motion, planet changes its direction and the tangential force should be decreased. As a result, Newton was forced to prescribe it to supernatural force: "Gravity might give the planets a motion of descent towards the Sun... yet the transverse motion, by which they revolve in their several orbs, required the divine arm" (Newton, Four letters, 1755, p. 34). Later the same problem appeared before quantum mechanics. Bohr (1970) was forced to postulate that there are some "energetic" orbs, where the electrons do not lose their energy (Bohr, 1970, p. 161; p. 452) -in spite of the known physical laws.

In the middle of XIX century, the notion of ether was resurrected due to the developing of wave optics, but towards the end of the century, it was refuted by the experiment of Michelson-Morley. The conceptual crisis put into the forefront the notion of the physical field that is nothing more than a space, possessing an ability to conduct and to contain the forces of remote action.

The "impure" remote action, proceeding through resilient material media, is well known. Male flowers of Vallisneria are detaching from a stem, floating on the surface of water, and attracting the greater sessile female ones; the attraction through the water may lead ships to collision. The kinds of remote action are the force of buoyancy, repulsing light objects from Earth through water and air (balloon); atmospheric and water pressure, participating in the gravity - in contrast of gas pressure in the tank. Small bubbles of cavitation may turn around greater ones by stable orbits (Kornfeld, 1951, p. 94). At least, the constancy of contemplator solid bodies is, in fact, dynamic one. Their shape is reconstituted through the orbital motion of electrons; their remote action with nuclei through the "field". Nevertheless, interaction of macroobjects (e.g., remote gears of a single mechanism) belongs to the closed action.

As a result of the remote action via a medium, repulsion occurs in the interval between the objects. The "attraction" (approaching) comes at the expense of pushing by medium behind the objects. The balance of these forces is the premise for a stable orbital movement that cannot be reduced to any of the given types of interaction. 
Perhaps, because the totality of matter cannot be shifted, motion proceeds in closed trajectories, forming circulating flows. I.e., the orbital motion is defined by the movement of all the totality.

The solidity of bodies in the absence of emptiness is defined by flows of a thin medium between electrons and nuclei. I.e., the solidity is dynamic characteristic. Common water, parted before swimmer, will appear a kind of solid body for an unlucky diver falling in it by his stomach.

The principle of dynamic nature of solidity will play a key role in the theory proposed.

In such a way, the remote action, as an establishment of connection between remote objects and construction of objects of greater level, in contrast to flows, is irreducible to physical mechanism and is rooted in its philosophic grounds. Physicists separated the remote action from spontaneous interactions in an artificial way via introduction of quasi-physical medium or the so-called hidden qualities of objects.

Understanding the basic role of the remote action permits to clear its mechanism from mystics.

\section{Theory of Tides}

During interaction through material medium, force of repulsion applies on the surface of the Earth immediately under the Moon. At the opposite side (in the nadir), the zone of pushing of the Earth with the Moon must be applied. It seems that water should pour off these localities...

Such a conclusion came from Descartes by proceeding from the remote action through the medium (Descartes, 1989, p. 226). The successive observation disproved his views. Nevertheless, in the anniversary of the death of Newton, i.e., 41 years after the issue of his "Mathematical Principles" and 100 years-after the publication of the "Treatise" of Descartes, Voltaire (1988) witnessed: "The deal comes to such a point that when you /Frenchman/ think that the Moon should be the cause of tide, those sirs /Englishmen/ assume, in contrary, that the ebb takes place" (Voltaire, 1988, p. 130).

Important corrections to the Newtonian static theory of tides are introduced by Laplace (1982). According to his dynamic theory, distribution of tides depends on the latitude of locality that determines the inertia of water. Nevertheless, for the general consideration, the theory of Newton is admissible, especially because it is diametrically opposite to the conclusion, following from the remote action via material media.

In the first approach, it could be noted that from the beginning the translation of gravity was assumed to be instantaneous: Descartes proceeded from incompressible basic medium (Descartes, 1989, p. 238), Newton-from the immediate remote action (Newton, Essentials, 1989, p. 468; p. 484). Adjusting the latter statement with the theory of relativity, Einstein (1965) limited distribution of gravity by the speed of light ${ }^{2}$ (Einstein, 1965, p. 504) that should imply delay of gravity from Moon on a few seconds and that from Sun—on a few minutes.

Meanwhile, the thinner is the resilient medium and the slower is progress of oscillations as the result of their dispersion. Thus, the velocity of sound in water is lesser than in metal and greater than in air. Correspondingly, the speed of gravity should be sufficiently lesser than that of sound in atmosphere. I.e., the water runs away from delayed influence of the Moon.

\footnotetext{
${ }^{2}$ Acceptance of light, in particular, of radiowaves for oscillations of ether and afterwards - electromagnetic field favored to such viewpoint. The analysis of the given thesis is out of the current article frames. I would notice only that neither visible light nor radiowaves do participate in the remote action proper. Furthermore, as "fields" occupy the greater part of the atom, the speed of sound may be also referred to transfer of their tension. The question about the nature of radiowaves is remained for further discussion.
} 
Indeed, the syzygia and quadratures ${ }^{3}$ - tides, where interaction between the Sun and the Moon is the most prominent, are delayed on 36 hours (Laplace, 1982, pp. 196-197). Daniel Bernoulli in the "Memoir for the theory of tides" explained this phenomenon by the delay of the force of gravity (cited from Laplace, 1982, p. 196). In his answer, Laplace pointed that if the speed of gravity is not infinite one, than in millions times greater than the speed of light (Laplace, 1982, pp. 196-197; p. 309). In such a way, the scientist defended the concept from the fact contradicting it.

Having no possibility to become acquaint with the "Memoir" of Bernoulli, I have no advice, which in terms of delay he put at the groundings of his concept. It may be said only that the interval of 36 hours seems too small and the syzygia and quadratures - idealization, following from the straight match of the phenomena. Pass of the distance about $150 \mathrm{mln}$. kilometers during 36 hours or $100 \mathrm{mln}$. $\mathrm{kms}$ per 24 hour $(\approx 4.2 \mathrm{mln}$. $\mathrm{km} / \mathrm{h} \approx 1,200 \mathrm{~km} / \mathrm{s})$ would appear in 3,600 times swifter than that of sound in atmosphere.

From the proportion mentioned, it may be obtained that if the correspondent interval were completely filled by the quite air with the normal temperature and pressure, sound would come from the Sun to the Earth during $3600 \times 36=129600$ hours, i.e., near $12 \frac{1}{3}$ years, and from the Moon to the Earth—during about 40 hours. Because the vacuum does not transmit the heard sound, hence, the dispersion of oscillation is sufficiently greater, and their speed - lower, syzigia and quadratures should be caused by even ancient position of the Sun and Moon. I.e., the delay of tides provides the empirical evidence of the interplanet space filled by resilient medium, possessing normal properties.

In the case of immediate influence of the Moon on the water ocean, the same should be waited for more closed and less inert aerial ocean — as Descartes supposed (Descartes, 1989, p. 226). Laplace found that the tides of atmosphere are of negligible value $-1 / 18 \mathrm{~mm}$ (Laplace, 1982, p. 210) ${ }^{4}$. Nevertheless, the air should not only be influenced by lunar force of gravity, but also to conduct it to the ocean. I.e., to form air currents is sufficient to draw the water. In this case, not tides, but storms, connected with Moon influence were observed. If tidal currents in air are dispersed by any extraneous factor, this would imply that the Moon influence is violated by the way towards the water and would not be able to rice oceanic tides.

Slow gravitational waves of resilient medium would not pierce atmosphere, like radiation, but collide with it, like with firmament as the result of the fast rotation of the globe, reacting with them as the comprehensive whole. Here, in contrast to the static gravitation, alteration of resilient joggings and pushes occurs. The oscillations

\footnotetext{
${ }^{3}$ Maximal and minimal tides, matched with the stages of the new and whole Moon, connected with the opposition of Sun and Moon - from one side; and the first and later quarter of the Moon, appeared by its divergence with the Sun at $90^{\circ}$ by the celestial arc-from the other side.

${ }^{4}$ The famous evolutionist, Lamarck, proceeding from the Newtonian theory of tides, made conclusion about existence of tidal currents in atmosphere and their influence on weather. In 1800 Ministry of the Interior of France collected meteorological data for their interpretation by Lamarck. The biographer of Lamarck writes: "Meanwhile the forecasts-as would be expected, unsuccessful, delivered to Lamarck many troubles... Laplace told about them with complete disregard... many men already begun look at the author as a charlatan... but Lamarck continued to publish the collection persistently up to 1810". In this year, Napoleon rebuked the aged scientist for his meteorological studies (Karpov, 1935, pp. CXX-CXXII). Such misfortunes cost many successes.

In the current physics of atmosphere, the influence of the Moon, as far as I know, is not considered. Thus, in the monography of Hrgian among the forces, influencing on atmosphere there are mentioned the force of gravity and the gradient of pressure; as collateral ones - declining force of the Earth rotation, centrifugal force by curvilinear motion of the air particles, the force of internal friction, the turbulent force and also - the magnetic-hydrodynamic force (Hrgian, 1969, p. 395), but not the tidal force.
} 
introduced are dispersed in the lesser degree in the firm cortex of Earth, from where they come to the water. Hence, tides are engendered by microseisms. As destructive earthquakes do not cause hurricanes, microseisms are not accompanied with winds. They are extinguished by vast intermolecular intervals in air that efficiently disperse influences.

In 1980-th, the group of Soviet scientists found that tides are connected with microseisms of high frequency. Nevertheless, coming from the Newtonian concept, the authors considered the phenomenon as consequence and not the cause of shifts of water masses ${ }^{5}$ (Rykunov, Havroshkin, \& Tsyplakov, ${ }^{6} 1980$ ).

The similarity between the tides and tsunami appears not convergent, connected with great length of wave, but, rather, genetic one. Taking into account relative safety, regularity and extensiveness of tides, the establishment of their seismic nature may introduce considerable contribution in the study of tsunami and forecast of loss from them.

\section{Theory of the Forced Seismic Waves}

Hence, the tides are introduced by earthquake, i.e., by pushes, causing ebbs. At the first view, such a conclusion contradicts to arrangement of tides, where maximum is found immediately below the Moon and on the opposite side of the Earth, i.e., on the maximal distance from epicenters. To this contradiction, the other one may be added: It is considered that the proper seismic waves are free ones, i.e., going by inertia after the push of an earthquake (Zhukov, 1976, p. 172). In this case, their energy would disperse in the open ocean than in the relatively small basins of the closed Mediterranean seas.

Meanwhile the opposite is true. The earthquakes proceeded near the Black Sea shore of Turkey that continued from 26 of December 1939 till 2 January 1940, which caused rise of water on $20 \mathrm{~m}$ in the town Fats (Turkey), came to the rocky shores of Novorossiysk and Yalta (Russia) as seismic waves of 53.4 and 14-cm height, correspondingly (Shniukov, Mitin, \& Tsemko, 1994, p. 9). During the destructive earthquake on the Black Sea shore of Turkey on 17 of August 1999, there were no tsunami, though the seismic pushes have been reached the opposite, Northern coast of the Black Sea, and were sensible in Kishinev. It is necessary to remark that vertical shifts predominated on horizontal ones; i.e., the earthquake was potentially tsunamigenic. At the same time, oceanic tsunami passed repeatedly from shores of Kamchatka, North and South America to Hawaii, New Zealand, and Australia and preserved their destructive ability (Bolt, 1981, p. 93). It may be assumed that, indeed, non-correspondence of shift scales, however, in the closed seas the display of microseismic waves, i.e., tides, is also extremely weak.

Even more clear non-correspondence to hypothesis of free waves is concluded in the assumption that tsunami spread over the entire water column. At the same time in the open ocean, they do not affect ships ${ }^{7}$. Small breaking force of the wave in the open ocean is explained by its low height (number of meters by kilometric lengthes) (Zhukov, 1976, p. 152). Nevertheless, getting in the current and running with the speed of 400-800 $\mathrm{km} / \mathrm{h}$, ships would become toy of the elements and the quiet air would act on the overwater part as a heavy-duty

\footnotetext{
${ }^{5}$ Both from Newtonian concept, it follows that alternating attraction of different portions of the earthen surface should cause seismic waves. Correspondingly, the income of "tidal perturbances" of Earth in the moonquakes is well known (Shevchenko, 1983, p. 36).

6 I do not mark the co-authors as "et al." since any of them is a person, who has made its own contribution to the research.

7 At the same time, it is recognized that acoustic waves from tsunami are able to stop a vessel (Zhukov, 1976, p. 151).
} 
hurricane (the speed of a common hurricane is in 2-4 times lesser). In addition, colliding with unagitated water (in fact, foreign liquid), seismic wave would shut up an abrupt and high billow, like during common storms.

In the given case, to author's mind, the shortcoming of experimental approach is clearly displayed: The habitual absence of natural phenomena does not comprehend, even by contradiction to the existing concept. One of the causes of such a simplification is the difficulty of object's study, complicated by numerous local factors, from which, in the general case we have to abstract.

The solution is seen in that the collision of agitated water with quiet one proceeds not by front, but at the depth. It explains the absence of the tsunami-caused shipwreck in the open ocean. Probably, here, like in the case of termocline, group inertia of water molecules detains considerable part of agitation, giving rise to dynamical leap. As a result, it is prevented dispersion of the seismic energy by vertical. Oscillations of the Earth cortex, spreading faster than those of water, eliminate the same resistance in horizontal direction.

In such a way, seismic waves are not free, but forced, therefore, rising as the result of resonance - comp. with wind oceanic waves that are much higher than the marine. Correspondingly, the oceanic bottom is more spacious than that of a sea. Furthermore, in the batial zone of ocean, the bottom is built from bedrock that conducts oscillations better than continental and sedimentary rocks of the shelf and marine bed.

It should be mentioned also that the speed of radial projection of Moon by the Earthen surface at the equator forms about 1,666.7 km/h and near Murmansk (on 69 th parallel-about $600 \mathrm{~km} / \mathrm{h}^{8}$. Correspondingly, speed of the tidal currents would exceed that of tsunami. Even taking into consideration water friction with Earth and atmosphere, uninterrupted gravitational wind, blowing billion years, would accelerate water up to hurricane speed with all the consequences. If tides are caused by microseisms, then oscillations of both, lithosphere and atmosphere are not only attracted together (the predominant process), but partially extinguish one another. As a result, the speed of the lunar epicenters corresponds to that of the tidal front, but not to the tidal currents. Otherwise, use of the deep apparatus became impossible, - not to mention that tidal waves would become stronger than tsunami.

Proceeding from the hypothesis of free waves and classical hydrodynamics, the theory of tsunami, contemporary to the author, is abstracted from both oscillation of bottom outside the epicenter and compression of water, assuming them insignificant (Burymskaya, 1983, p. 3). If seismic waves are forced, the given factors, in contrary, play the key role.

Consequently, in contrast to the accepted version, the tsunami could not embrace the entire water column evenly. From the gradient of velocities, it follows that the surface of ocean cannot react at the pass of a deep seismic wave simultaneously. Furthermore, the deep waves, in accordance with their greater speed, should be shorter and higher than superficial ones. Hence on the depth should appear zones of water compression, increasing in addition potential energy of the seismic wave and kinetic one - of the tsunami proper.

The compression of water that may be neglected in hydraulic, in the oceanology is an important factor. It may be defined by the formula: $\Delta \mathrm{H}=\frac{\mu P}{2} H=\frac{\mu P^{2}}{2}=\frac{\mu H^{2}}{2}$ (in equated units: decibars/meters) where $P$ is

\footnotetext{
${ }^{8}$ The accepted formula for length of a parallel calculation is: $l=2 \pi \mathrm{R} \cos \alpha$, where $\alpha$ is the degree of latitude, $\mathrm{R}$-radius of the Earth (Zhukov, 1976, p. 170). However, since the latter equals to L: $2 \pi$, where $\mathrm{L}$ is the length of the circle with the given radius $(\approx$ the length of the equator), irrational, and hence inconvenient coefficient $2 \pi$ may be canceled, exchanging the radius of the Earth by the length of the equator. In addition we can abbreviate both the length of equator $40,000 \mathrm{~km}$, and the time of the Moon rotation $\approx 24 \mathrm{~h}$ in 4 times. Hence, $10000 \cos 69^{\circ}(\approx 0.374606): 6 \mathrm{~h}=624 \mathrm{~km} / \mathrm{h}$.
} 
water pressure; $H$-depth; $\mu-$ middle coefficient of the water compression, etc., equal to $4,254 \times 10^{-9}$ decibars ${ }^{-1}$ by the salinity of $35 \%$ and temperature of $5{ }^{\circ} \mathrm{C}$ (Zhukov, 1976, p. 79). Hence for the middle oceanic depth of $4 \mathrm{~km}$ the compression forms about $34 \mathrm{~m}$. Taking (in accordance with averaged data for tsunamigenic earthquakes of the Pacific ocean (Burymskaya, p. 61) depth of the focus for $40 \mathrm{~km}$, magnitude for 7.3 that corresponds the earthquakes of eight points or superficial acceleration about $2 \mathrm{~m} / \mathrm{s}^{2}$ (Nazarov \& Darbinian, 1974, p. 27), i.e., about $1 / 5 \mathrm{~g}$, and density of basalt, equal to three water densities, the seismic compression exceeds that of oceanic in six times.

If the pushes of such an earthquake were spread evenly by the water column of 4 kilometers, they could compress it in addition on $184 \mathrm{~m}$. Nevertheless, as was said above, barocline should hinder agitation's spread. Even if compression embraces few dozens of feet, it is sufficient for hindering of a seismic wave. Perhaps, this value corresponds to the ebb in the epicenter of earthquakes whether tidal microseism.

The significant factor, influencing on the course of seismic waves, seems to be the relief of batial. Its considerable changes or alteration of epicenter position are in particular importance for the forecast of loss from tsunami. The significance of tide was mentioned above.

\section{Conclusion}

The nature of tides is seismic and they should be studied as a kind of tsunami. Tsunami are forced waves, feeding during their distribution by oscillations of earthquakes and spreading in the open ocean down the layer of non-accelerated water within the seismic compression of a water column.

\section{References}

Bohr, N. (1970). Selected scientific works in two volumes (Vol. 1): About the spectrum of hydrogen (pp. 152-167); the structure of atom (pp. 417-452). Moscow, Nauka.

Bolt, B. (1981). Earthquakes, popular essay. Moscow: Mir.

Burymskaya, V. N. (1983). Kinematic and dynamic of tsunamigenic earthquakes foci. Vladivostok: Far Eastern Scientific Center of Ac. Sci. of USSR.

Democritus. (1970). Texts, translation, investigations, composition and translation by S. Ya. Lurie, Leningrad: Nauka.

Descartes, R. (1989). Works in 2 volumes (Vol. 1): The world or treatise about the light. Moscow: Mysl.

Dirac, P. A. M. (1933). Theory of electrons and positrons. Retrieved December 12, 1933 from http://www.nobelprize.org/nobel_prizes/physics/laureates/1933/dirac-lecture.pdf

Einstein, A. (1965). Collection of scientific works in four volumes (Vol. 1): Essentials of general theory of relativity (pp. 452-504). Moscow: Nauka.

Hrgian, A. H. (1969). Physics of atmosphere. Leningrad: Gidrometgiz.

Karpov, V. P., \& Lamarck, J. B. (1935). A biographical essay (pp. XCIX-CLIII): Philosophy of zoology. Moscow-Leningrad: State Publisher of Medical and Biological Literature.

Kornfeld, M. I. (1951). Firmness and resilience of liquids. Moscow-Leningrad: State Publisher of Technic-Theoretic Literature.

Laplace, P. S. (1982). Exposition of the system of the world. Moscow: Nauka.

Nazarov, A. G., \& Darbinian, S. S. (1974). Essential of a quantative estimation of strong earthquakes intencity. Yerevan: Ac. of Sci. of Armenian SSR Press.

Newton, I. (1755). Four letters from Sir Isaak Newton to Doctor Bentley, containing some arguments in proof of a deity. London: Printed for R. and J. Dodsley, Pall Mall MDCCLV.

Newton, I. (1952). Opticks or a treatise of the reflections, refractions, inflections \& colours of light. NY: Dover Publications Inc.

Newton, I. (1989). Mathematical principles of natural philosophy. Moscow: Nauka.

Rykunov, L. N., Havroshkin, O. B., \& Tsyplakov, V. V. (1980). Lunar-solar tidal periodicity in spectrum lines of high-frequency microseisms temporal variation. Reports of Ac. Sci. of USSR, 252(3), 557-580. 
Shevchenko, V. V. (1983). The moon and its observation. Moscow: Nauka.

Shniukov, E. F., Mitin, L. I., \& Tsemko, V. P. (1994). Catastrophes in the Black Sea. Kiev: Manuscript.

Voltaire, F. M. A. (1988). Philosophical works, philosophical letters (pp. 70-226): Letter fourteen, about Descartes and Newton (pp. 130-134). Moscow: Nauka.

Zhukov, L. A. (1976). General oceanology. Leningrad: Gidrometeoizdat. 